\title{
The importance of tacrolimus in the treatment of allergic keratoconjunctivitis
}

\author{
Georgia Araujo*, Mariana Nascimento, Patricia Arruda, Luiz Felipe Moraes, Marina De Andrada Carvalho, \\ Décio Medeiros, Emanuel Sarinho \\ From 3rd WAO International Scientific Conference (WISC) 2014 \\ Rio de Janeiro, Brazil. 6-9 December 2014
}

\section{Background}

Keratoconjunctivitis (KC) is an eye disease that potentially leads to blindness if not treated properly, which often requires follow-up for more than an expert. The aim of this study is to evaluate the response of topical tacrolimus in allergic keratoconjunctivitis.

\section{Methods}

A longitudinal study of series of cases conducted between September/2013 and June/2014, with 24 patients followed in Ocular Allergy ambulatory, Hospital das Clinicas of the Federal University of Pernambuco. The patients included in the study had symptoms such as: itching, burning, redness and/or edema uncontrolled medical monitoring initial and were then taken to the ophthalmologist for defining more specific treatment.

\section{Results}

The median age was 10 years, with 15 male patients (62\%). Of the 24 patients, 14 (58\%) patients had allergic rhinitis, 7 (29\%) had a diagnosis of asthma and rhinitis associated and $2(8 \%)$ had rhinitis, asthma and atopic dermatitis. All were using medications for rhinitis and/ or asthma continuously. Regarding the treatment of conjunctivitis, only 1 patient (4\%) remitted symptoms using of mast cell membrane stabilizer, $6(25 \%)$ have control of symptoms $\mathrm{KC}$ using mast cell membrane stabilizer and antihistamine, others 6 (25\%) was necessary to add an ocular lubricant. In 11 patients (46\%) with severe allergic $\mathrm{KC}$ was necessary to associate a topic corticosteroids and 5 (45\%) of these group became steroid-dependent, only reaching remission of symptoms with tacrolimus $0.03 \%$ ophthalmic ointment. All patients who used tacrolimus reported improvement in signs and symptoms and adverse events were limited to the local burning in one patient who interrupted treatment, despite that improvement of edema and hyperemia.

\section{Conclusions}

Allergic rhinitis is very common in patients with $\mathrm{KC}$, underscoring the importance of joint processing for clinical improvement. The use of topical tacrolimus $0.03 \%$ in and out of the conjunctival sac seems to be in the short term, effective, well tolerated and safe in the treatment of allergic conjunctivitis refractory to traditional treatment, and avoid prolonged corticosteroid therapy and its associated side effects, such as hypertension eyepiece.

\section{Published: 8 April 2015}

\section{References}

1. Chen JJ, Applebaum DS, Sun GS: Atopic keratoconjunctivitis: A review. Am Acad Dermatol SC 2014, 70(3):569-75.

2. Erdinest N, Solomon A: Topical immunomodulators in the management of allergic eye diseases. Curr Opin Allergy Clin Immunol 2014, 21.

doi:10.1186/1939-4551-8-S1-A234

Cite this article as: Araujo et al:: The importance of tacrolimus in the treatment of allergic keratoconjunctivitis. World Allergy Organization Journal 2015 8(Suppl 1):A234. 